Full Length Article

\title{
Magnetically-extractable hybrid of magnetite, mesoporous silica and titania for the photo-degradation of organic compounds in water
}

\author{
Alexandre de Oliveira Jorgetto ${ }^{\mathrm{a}, \mathrm{b}, *}$, Alexander Milbrat ${ }^{\mathrm{b}}$, José Fabián Schneider ${ }^{\mathrm{c}}$, Zheng Li $^{\mathrm{b}}$, \\ Guido Giammaria $^{\mathrm{d}}$, Margarida Juri Saeki ${ }^{\mathrm{a}}$, Thiago Marcelo Ribeiro Gianeti ${ }^{\mathrm{e}}$, \\ Giuseppina Pace Pereira Lima ${ }^{a}$, Valber de Albuquerque Pedrosa ${ }^{a}$, Guido Mul ${ }^{b}$, \\ Gustavo Rocha de Castro ${ }^{\mathrm{a}}$ \\ ${ }^{a}$ Universidade Estadual Paulista/UNESP, Departamento de Química e Bioquímica - Instituto de Biociências (IB), Caixa Postal 510, 18618-000 Botucatu, SP, Brazil \\ ${ }^{\mathrm{b}}$ Photocatalytic Synthesis Group, MESA + Institute for Nanotechnology, Faculty of Science and Technology, University of Twente, Meander 229, P.O. Box 217, 7500 AE \\ Enschede, The Netherlands \\ ${ }^{\mathrm{c}}$ Instituto de Física de São Carlos (IFSC), Universidade de São Paulo (USP), CEP 13566-590 São Carlos, SP, Brazil \\ ${ }^{\mathrm{d}}$ Catalytic Processes and Materials, MESA +, Faculty of Science and Technology, University of Twente, P.O. Box 217, 7500AE Enschede, The Netherlands \\ e Universidade Estadual Paulista/UNESP, Laboratório Central - Faculdade de Ciências Agronômicas (FCA), Caixa Postal 1780, 18610-307 Botucatu, SP, Brazil
}

\section{A R T I C L E I N F O}

\section{Keywords:}

MCM-41 silica

Titania coating

Photocatalysis

Magnetic extraction

Anatase crystal growth

Advanced oxidation process

\begin{abstract}
A B S T R A C T
This work addresses the development of a magnetically extractable magnetite-silica-titania photocatalyst to be applied in the degradation of organic compounds in water. MCM-41 silica was successfully deposited on magnetite, providing large surface area for anchoring of $\mathrm{TiO}_{\mathrm{x}}$ species, and preventing thermally induced conversion of magnetite to hematite. A good correlation between the calculated values and amount of titania deposited on the silica-covered magnetite particles was obtained for a synthesis route involving the treatment of magnetitesilica in boiling ethanolic Ti-precursor solution. Photocatalytic activity in conversion of 4-chlorophenol could only be observed for compositions containing larger than $\sim 50 \mathrm{wt} \%$ titania, whereas increasing the titania content did not significantly improve performance. Experiments carried out at $\mathrm{pH} \sim 3.0$ and $\sim 7.2$ demonstrated the performance is relatively $\mathrm{pH}$-independent. The structure activity-correlation of the materials is briefly discussed.
\end{abstract}

\section{Introduction}

The increasing diversity and concentration of toxic components in water resources requires efficient technology for purification. Several organic pollutants are persistent and not easily degraded through conventional bio-based methods [1-3]. Heterogeneous photocatalysis, utilizing slurries of titanium dioxide and UV radiation in aqueous media, has been demonstrated a very effective alternative technology [4]. Reactive oxygen species (ros) are formed by reaction of the photoactivated $\mathrm{TiO}_{2}$-based photocatalyst with oxygen (reductively, yielding super-oxide anions), and water (oxidatively, yielding hydroxyl radicals), respectively. Once formed, ros convert organic substances to $\mathrm{CO}_{2}$ and $\mathrm{H}_{2} \mathrm{O}$ [3-5].
Until recently, difficulties in the separation of particulate photocatalysts through conventional methods (e.g. filtration and centrifugation) still have posed serious limitations on their application in largescale chemical processes. Consequently, the full potential of their active surface area has not been fully exploited at industrial scales. In an attempt to solve such issue, magnetic titania-based particulate photocatalysts have been developed in the last decades, aiming the incorporation of magnetic properties to these photocatalysts to enable a practical and cost-effective strategy for particle retrieval and separation from slurries [6-11]. Core-shell structure materials proved to enable efficient particle separation, and different types of ferrites have been commonly used to constitute their cores because of their simple syntheses, low cost, low toxicity and controllable magnetic properties

Abbreviations: $M$, magnetite; $M(c)$, calcined magnetite; $S(c)$, calcined MCM-41 silica; $M S(c)$, calcined silica-coated magnetite; $M S T$-\#, magnetite-silica-titania composite containing \#

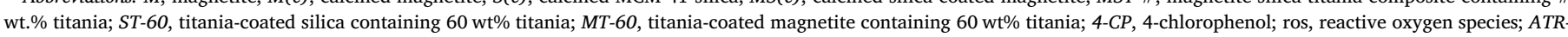

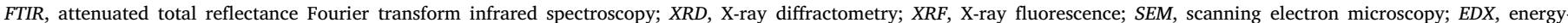
dispersive X-ray spectroscopy; CTAB, cetyltrimethylammonium bromide; TEM, transmission electron microscopy; TEOS, tetraethylorthosilicate; TIP, titanium isopropoxide

* Corresponding author at: Universidade Estadual Paulista/UNESP, Departamento de Química e Bioquímica - Instituto de Biociências (IB), Caixa Postal 510, 18618-000 Botucatu, SP, Brazil.

E-mail address: xjorgetto@gmail.com (A.d.O. Jorgetto). 
through doping with other elements $[10,11]$.

Photocatalysts with titania deposited directly on ferrites present drawbacks related to photodissolution of the cores of such materials and the alteration of the electronic properties of titania, however these effects were effectively circumvented by the incorporation of an intermediary silica layer between the magnetic core and the titania layer in such materials [6,8-13]. Silica intermediary layer was also found to play a crucial role on the phase preservation of ferrite cores of the hybrid materials by not allowing them to convert to less magnetic iron oxides phases $[6,10]$. Despite the deposition of continuous silica has been commonly described, not much is reported on the use of mesoporous silica for magnetically-extractable photocatalysts, being the main interest in the application of mesoporous silica to increment significantly the surface area of the photocatalysts and therefore its photocatalytic efficiency.

This paper addresses the issue of separation/immobilization of the catalyst by the synthesis of a magnetite-silica-titania composite, allowing magnetic extraction of the particles from the liquid media. A mesoporous silica-based interface between titania and magnetite was deposited to prevent conversion of magnetite to hematite upon thermal treatment as well as the leaching of $\mathrm{Fe}^{2+/ 3+}$ in solution, and at the same time to provide a high surface area (which for $\mathrm{TiO}_{2}$ is typically less than $100 \mathrm{~m}^{2} \mathrm{~g}^{-1}$ [5]), potentially contributing to efficient generation of ros and pollutant conversion. MCM-41-type porous silica was employed to attain mesopores and surface areas higher than $1000 \mathrm{~m}^{2} \mathrm{~g}^{-1}$ [14]. A titania coating step, involving the treatment of magnetite-silica in boiling ethanolic precursor solution was developed, and the obtained materials were characterized through techniques such as ATR-FTIR, XRD, XRF, Raman spectroscopy, SEM, EDX, TEM and surface area measurements. 4-chlorophenol was used as a target substrate to evaluate the photocatalytic performance of the developed materials for the decomposition of recalcitrant organics. The effect of various process parameters was evaluated, such as $\mathrm{pH}$, substrate concentration, and catalyst concentration.

\section{Experimental}

\subsection{Synthesis of the mesoporous silica-covered magnetite}

$6.0 \mathrm{~g}$ of $\mathrm{FeCl}_{3}$ (Sigma-Aldrich, 97\%) was dissolved in $75.0 \mathrm{~mL}$ of dionized water. In another vessel, $6.0 \mathrm{~g}$ of $\mathrm{FeCl}_{2} \cdot 4 \mathrm{H}_{2} \mathrm{O}$ (SigmaAldrich, > 99\%) was dissolved in $35.0 \mathrm{~mL}$ of de-ionized water. Under nitrogen atmosphere, both solutions were mixed together into a $250 \mathrm{~mL}$ Erlenmeyer flask, and the final mixture was heated to $80^{\circ} \mathrm{C}$. Afterwards, $50.0 \mathrm{~mL}$ of $\mathrm{NH}_{4} \mathrm{OH}$ (Dinâmica, 29\%) was added and the mixture was kept agitated for 30 min using a magnetic stirrer. By applying a magnetic field (with the aid of a rare-earth magnet) at the bottom of the vessel for some minutes (from outside the flask), the magnetite could be easily decanted and retained inside the reaction vessel, while the supernatant was removed (the magnet should be as large as the bottom of the Erlenmeyer for an effective decantation). Approximately $100 \mathrm{~mL}$ of deionized water were added to the flask with magnetite, which was again stirred to remove residual reactants and then the magnetic decantation and retention of the magnetite were repeated. The washing step with deionized water was repeated two more times, and then the magnetite was stored in water, whose colloidal concentration was determined to $34.0 \mathrm{mg}$ of magnetite per milliliter of solution. (Every time magnetite had to be synthesized, the colloidal concentration of the final mixture could be easily adjusted to the desired concentration by magnetically decanting the magnetite and removing or adding water to the flask). Part of the synthesized magnetite was calcined at $600{ }^{\circ} \mathrm{C}$ for $4 \mathrm{~h}$. The bare magnetite and the calcined magnetite are named throughout the text as $M$ and $M(c)$, respectively. The silica-covered magnetite particles were prepared in a mixture containing $200.0 \mathrm{~mL}$ of $\mathrm{NH}_{4} \mathrm{OH}$ $(29 \% \mathrm{w} / \mathrm{w})$ and $250.0 \mathrm{~mL}$ of deionized water, to which $2.0 \mathrm{~g}$ of cetyltrimethylammonium bromide $(C T A B)$ was added. The mixture was kept under agitation at $30^{\circ} \mathrm{C}$ until complete dissolution of the surfactant. Afterwards, $20.0 \mathrm{~mL}$ of the magnetite colloidal solution were added to the reaction mixture, followed by $10.0 \mathrm{~mL}$ of tetraethylorthosilicate (TEOS) (Sigma-Aldrich, > 99\%). The mixture underwent agitation for $2 \mathrm{~h}$, and then was filtered through a Büchner funnel. The reaction produced a grey powder, which was stored in a heated chamber at $45^{\circ} \mathrm{C}$ overnight to remove the solvent. The dry material was softly minced and then was also calcined at $600{ }^{\circ} \mathrm{C}$ for $4 \mathrm{~h}$. Bare silica was synthesized and calcined following the same steps, except that $270 \mathrm{~mL}$ of deionized water were used, and the addition of the magnetite solution was omitted. As the calcination step may cause the condensation of surface silanol groups, hydration was carried out by agitating $5 \mathrm{~g}$ of the silicabased materials in $100 \mathrm{~mL}$ of deionized water under reflux at $\sim 100^{\circ} \mathrm{C}$ (boiling water) for $4 \mathrm{~h}$. Afterwards the materials were dried at $70{ }^{\circ} \mathrm{C}$ overnight to remove excess of water. The materials produced in this step are called $S(c)$ and $M S(c)$ for the calcined silica and the magnetitesilica hybrid, respectively.

\subsection{Titania coating through ethanol boiling}

The material $M S(c)$ had its surface activated at $105^{\circ} \mathrm{C}$ in a drying oven for one hour at room pressure. Then $1.20 \mathrm{~g}$ was transferred to a beaker containing $80 \mathrm{~mL}$ of absolute ethanol (AA Chemie B.V., 99.8\%). The mixture was heated up to mild boiling $\left(\sim 80^{\circ} \mathrm{C}\right)$ under magnetic agitation, and as soon as the solvent started to boil, a certain volume of titanium isopropoxide (TIP) (Sigma-Aldrich, 97\%) was added. Heating was provided up to complete dryness of the mixture and a slurry was formed nearly at the end of the drying process, which required it to be manually revolved with the aid of a glass stick. Much care had to be taken at this point, once the mixture started 'popping', with evolution of a white gas. The obtained dry materials were softly milled and then taken to a calcination oven, in which they were calcined at $500{ }^{\circ} \mathrm{C}$ for $4 \mathrm{~h}$. The volumes of TIP evaluated in this study were $0.70,3.50,5.26$, 7.00 and $14.0 \mathrm{~mL}$, which stoichiometrically should provide approximately 13, 43, 53, 60 and 75\% in mass (considering that all the IPT was converted to titania, and that all the titania was deposited as a layer over the $M S(c)$ particles). These values will be used to refer to the materials along this work as follows MST-13, MST-43, MST-53, MST-60 and MST-75. Finally, a selection of the most efficiently magneticallyextractable particles was carried out prior to the degradation experiments, which consisted of stirring the materials in a $\mathrm{NaOH}$ solution at $\mathrm{pH} 10$ for $5 \mathrm{~h}(1.7 \mathrm{~g}$ in $100 \mathrm{~mL})$ and collecting the particles with the aid of a large rare-earth magnet wrapped in solution-impermeable plastic (the magnet was kept in the mixture for $10 \mathrm{~min}$ without stirring), and discarding the non-retained particles that remained in the mixture. After the stirring step with $\mathrm{NaOH}$ solution just mentioned, the material was also dispersed in deionized water and recollected with the magnet during $10 \mathrm{~min}$ in the same way as just described. This step was carried out a couple of times, and the particles not attached to the magnet within the established extraction time were discarded as well. Such process was carried out until there were virtually no particles dispersed in the aqueous medium after a treatment-time of $10 \mathrm{~min}$. The reason for stirring the material with $\mathrm{NaOH}$ solution at $\mathrm{pH} 10$ was to promote the disaggregation of clusters by charging their surfaces negatively. Alternatively, in order assess the contribution of each constituent of the composites in the degradation process, the route to obtain the material $M S T-60$ was also directly applied to bare magnetite $(M)$ and to the bare mesoporous silica $[S(c)$ ] as precursor materials [in contrast to $M S(c)$ ] to obtain, respectively, the materials $M T-60$ and $S T-60$, which also had their performances evaluated in terms of their initial degradation rates, for comparison.

\subsection{Characterization of the materials}

Infrared spectra of the samples were collected through ATR mode in a Perkin Elmer Spectrum 100 series spectrometer at a resolution of $4 \mathrm{~cm}^{-1}$. 

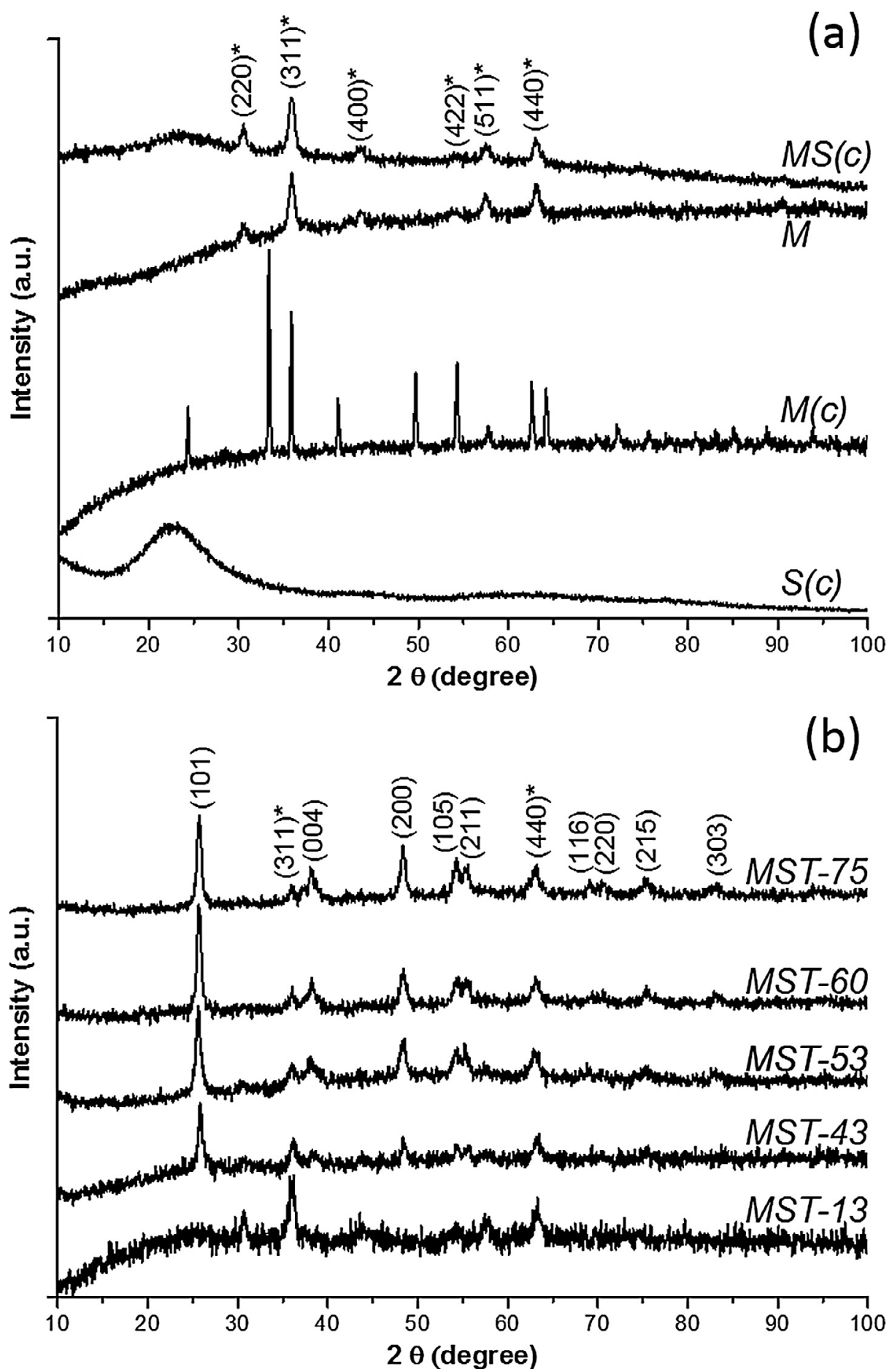

Fig. 1. XRD analyses for the single precursor materials $S(c), M, M(c)$ and $M S(c)$ (a) and for the titania-coated hybrid materials (b). Magnetite peaks are indicated by a * mark.

X-ray diffractograms were collected in a Bruker D2 phaser diffractometer in the $2 \theta$ range of $10-100^{\circ}$ using $\mathrm{Cu} \mathrm{Ka}$ radiation $(\lambda=0.15406 \mathrm{~nm})$. The $2 \theta$ increment was $0.051^{\circ}$, and the time to collect the signal at each step was $0.50 \mathrm{~s}$. The entire range took 1781 steps, and the voltage used was $30 \mathrm{kV}$. Raman analyses were carried out in a Bruker Senterra Microscope at a wavelength of $532 \mathrm{~nm}$, with an objective lens of $50 \times$ magnification. Scanning electron and EDX imaging were performed in a Zeiss Merlin HR-SEM, using an in-lens detector for SEM imaging and the materials were dispersed from ethanolic medium over a holey carbon film that was fixed onto Cu grids specially designed for electron microscopy analysis. Nitrogen adsorption measurements were collected in an ASAP 2400 Micromeritics instrument, and the specific surface areas of the materials were determined according to BET model. XRF analyses were carried out in a Bruker S8 Tiger and prior to analysis, the samples were fused at $1100{ }^{\circ} \mathrm{C}$ to produce beads which could reproducibly be measured. High-resolution ${ }^{29} \mathrm{Si}$ NMR experiments were performed at magnetic field of $9.4 \mathrm{~T}$ with a Varian Unity INOVA spectrometer. Samples were spun up to $5 \mathrm{kHz}$ in $7 \mathrm{~mm}$ zirconia rotors in the magic angle condition. The ${ }^{29} \mathrm{Si}$ spectra were obtained from single pulse experiments (direct polarization) using $\pi / 2$-pulse duration of $5.0 \mu \mathrm{s}$, recycle delay of $500 \mathrm{~s}$, and collecting up to 400 signals. A sample of tetrakis(trimethylsilyl)silane was used as secondary 
standard for the ${ }^{29} \mathrm{Si}$ chemical shift $(-9.9 \mathrm{ppm}$ relative to tetramethylsilane, TMS). Transmission Electron Microscopy (TEM) images were collected in a CM 200 Super Twin microscope (Philips). The electron beam acceleration potential was $200 \mathrm{kV}$, and the samples were prepared from ultradiluted suspensions of the materials, which were later deposited on an ultrathin carbon grid prior to the analysis. Tests to ascertain the decomposition of the hybrid materials in the suspensions were performed by means of Inductively-Coupled Plasma-Optical Emission Spectroscopy (ICP-OES) in an Optima 8300 equipment (Shimadzu). Calibration curves were prepared by diluting $1000 \mathrm{mg} \mathrm{L}^{-1}$ stock solutions of each element to adequate concentrations in three-component mixtures.

\subsection{Photodegradation experiments}

The activity of the hybrid materials for the degradation of organic compounds from water was evaluated by analyzing the conversion of 4chlorophenol (4-CP). The experiments were carried out by stirring the materials in $20.0 \mathrm{~mL}$ of a 4-CP solution of known concentration in two different regimes, namely, in the dark and under UV, which were carried out in a closed box reactor provided with internal magnetic stirrers. Although the magnetic particles were agitated by magnetic bars, the rotation of $600 \mathrm{RPM}$ proved to be more than sufficient to prevent their deposition on the surface of the magnetic bars. The reactors were positioned on a multiple stirrer plate, and enclosed in a box containing eight tubular light sources with a combined intensity of $3.2 \mathrm{~mW} \mathrm{~cm}^{-2}$ (in the wavelength range of $360-380 \mathrm{~nm}$ ). Parameters evaluated consisted of contact time (5-300 $\mathrm{min}), \mathrm{pH}(\sim 3.0$ and $\sim 7.2)$, catalyst concentration $\left(0.25-10.0 \mathrm{~g} \mathrm{~L}^{-1}\right)$ and concentration of $4-C P(\sim 16$ to $\sim 31 \mathrm{mg} \mathrm{L}^{-1}$ ), which were studied in a uni-variable mode. By the end of the established times for a given condition, $3 \mathrm{~mL}$ aliquots were collected and transferred to $15 \mathrm{~mL}$ Falcon tubes, which then underwent an external magnetic field provided by rare-earth magnets for $40 \mathrm{~min}$ for the displacement and retention of the particles inside the tubes. Afterwards, $2 \mathrm{~mL}$ sub-aliquots of supernatants were carefully collected and transferred to quartz cuvettes to have their concentration determined in a UV-Vis spectrometer (Thermo Scientific Evolution $600 \mathrm{UV}$-Vis) at the wavelength of $225 \mathrm{~nm}$. Prior to the photodegradation experiments, 4$C P$ solutions had synthetic air bubbled through them for $1 \mathrm{~h}$. In order to compare the efficiency of the synthesized composites with well-known commercial photocatalysts, the initial degradation rates obtained using Hombikat $100 \mathrm{UV}$ (Hombikat, Anatase phase) and Aeroxide ${ }^{\circledast} \mathrm{TiO}_{2}$ P 25 (P25, mixed Rutile and Anatase phases) were also determined for different catalyst concentrations. Non-magnetic materials were filtered through filter rings (Spartan 30/0.2 RC from GE Healthcare) before determination of the 4-CP concentrations. Photocatalytic experiments were carried out at conditioned room temperature of $20^{\circ} \mathrm{C}$. A typical degradation experiment resulted in a decrease in 4-CP concentration, which can be verified from Fig. S1 from the Supplementary Information).

\section{Results and discussion}

\subsection{Characterization of the materials}

\subsubsection{XRD and Raman analyses}

In order to investigate the crystal phases of $\mathrm{Fe}_{\mathrm{x}} \mathrm{O}_{\mathrm{y}}$ and of the obtained $\mathrm{TiO}_{2}$ in the as-synthesized materials, XRD analysis was carried out and the resulting diffraction patterns are shown in Fig. 1. The diffraction pattern of $M$ [in Fig. 1(a)], corresponds well to the magnetite phase (*), with the corresponding index planes indicated on top of the figure [15]. By comparing the magnetite diffraction pattern to that of calcined magnetite $[M(c)]$, a phase transition from magnetite to hematite $\alpha-\mathrm{Fe}_{2} \mathrm{O}_{3}$ is apparent, caused by the oxidation of $M\left(\mathrm{Fe}_{3} \mathrm{O}_{4}\right)$ during the calcination step. The difference in line-width of the magnetite (broad) and hematite (narrow) phases indicates that the magnetite structure is less crystalline in comparison to hematite, and may also imply that the magnetite crystallites are smaller than those of hematite. The transformation from magnetite to hematite is highly undesired (for the hybrid materials) because hematite presents very poor magnetization, which renders magnetic extraction unfeasible.

The diffraction pattern of as-synthesized silica [S(c) in Fig. 1(a)], only shows a broad feature ranging from 15 to $30^{\circ}$, typical of the mesoporous structure of MCM-41. Interestingly, the diffraction pattern of $M S$ (c) shows that the presence of the silica shell prevents phase transformation of magnetite to hematite during calcination. This may be explained by the inhibition of oxidation of magnetite by molecular oxygen, confirming that an effective coating of silica (MCM-41) over the magnetite particles has been achieved.

By comparison of the diffraction pattern of the titania-coated hybrid materials [Fig. 1(b)], all materials but MST-13 contain crystalline Anatase-phase titania (the corresponding index planes are indicated, the * indicating the magnetite phase). The diffraction pattern of MST-13 only contains the lines of magnetite, which indicates that probably the amount of titania deposited on this material was insufficient to give rise to titania crystallites. The diffraction pattern of the materials MT-60 and $S T-60$ were also collected and titania features were only observed for ST-60, whereas only magnetite features could be observed for MT-60 (see Fig. S2 of the Supplementary Information). This can imply that the direct deposition of titania over magnetite particles according to the here-proposed coating route, may not be effective.

Raman spectroscopy basically provides analogous results to those previously discussed for the XRD analysis. For instance, phase conversion from magnetite to hematite is obvious when magnetite particles are not coated by silica, and the lack of an Anatase diffraction pattern for the material MST-13 is in agreement with the absence of the Raman signature of Anatase in the spectrum of MST-13 (results are shown in Fig. S3 of the Supplementary Information). The presence of the Anatase phase could be confirmed for the other titania-coated materials, and the spectra may be found in Fig. 2.

\subsubsection{Infrared spectroscopy and XRF analysis}

The collected spectra of the titania-coated materials are shown in Fig. 3. Since all the materials yielded very similar spectra (see Fig. S4 of the Supplementary Information for full range spectra), only the spectral range in which the most remarkable differences were found is depicted. The most notable bands in the $1300-650 \mathrm{~cm}^{-1}$ region can be assigned to the vibrational modes of silica groups, and according to the literature [16], the Anatase phase of $\mathrm{TiO}_{2}$ contributes to spectral intensities around $650 \mathrm{~cm}^{-1}$, found at the spectral limit of the shown figure. The arrow indicated as (i), indicates a decrease in spectral intensity at

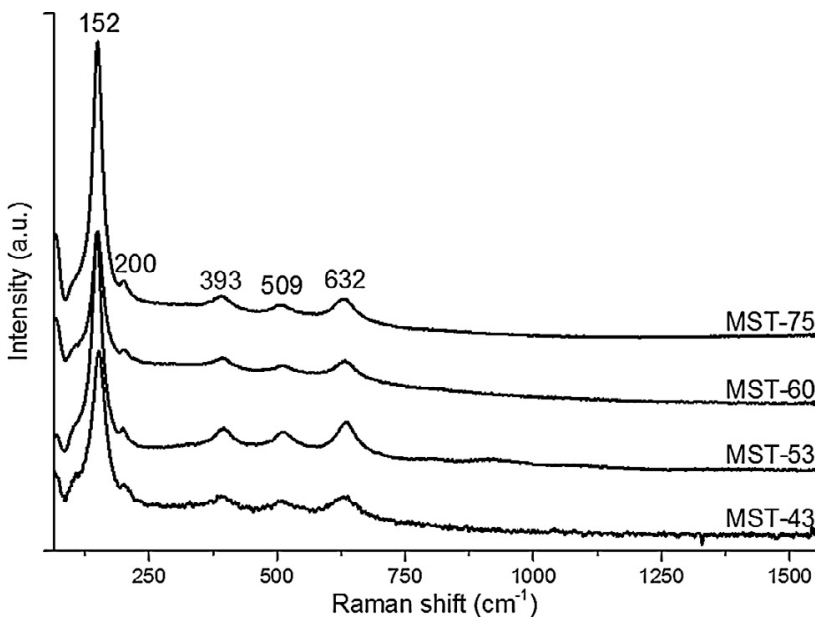

Fig. 2. Raman spectra of the titania-coated materials, clearly demonstrating the presence of Anatase phase in the samples MST-43 to MST-75. 


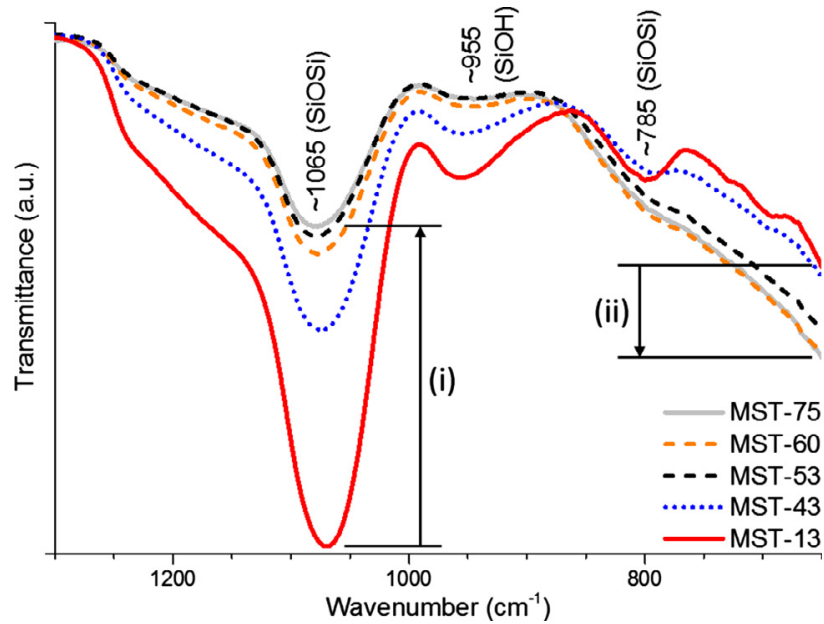

Fig. 3. Infrared ATR spectra of the hybrid materials with the assignments of the vibrational groups as indicated. All spectra are plotted without any adjustment in height and intensity, and all samples were analyzed through the ATR-FTIR technique applying the same pressure at the optical crystal (diamond).

$1065 \mathrm{~cm}^{-1}$ as a function of increasing titania content from the material MST-13 to the material MST-75. The band at $955 \mathrm{~cm}^{-1}$ shows a similar trend. In contrast, baseline transmittance around $650 \mathrm{~cm}^{-1}$ increases in intensity as indicated by arrow (ii), in agreement with the increasing $\mathrm{TiO}_{2}$ content. The spectral intensity changes indicate that silica is progressively covered with $\mathrm{TiO}_{2}$ moieties. Interestingly, the decrease in transmittance at $1065 \mathrm{~cm}^{-1}$ and increase of baseline transmittance at the extremity of the spectral range at $650 \mathrm{~cm}^{-1}$ are not linear, but trend towards a respective intensity limit. The materials MST-53, MST-60 and MST-75 showed nearly the same absorption intensities. This non-linearity is in agreement with Anatase crystallite growth at high loading, not requiring additional interaction with the silica surface, as demonstrated by the XRD and Raman analysis of the MST-53, MST-60 and MST-75 samples.

To confirm that the $\mathrm{TiO}_{2}$ loading is according to the amount of precursor introduced in the synthesis solution, XRF analysis was performed. The result of the analyses is depicted in Fig. 4(a), and not surprisingly indicates that iron oxide, silica and titania are the main constituents of the composite materials. An increase in the amount of titania is apparent, and the silica:magnetite ratio was nearly $2: 1$ in all cases. Fig. 4(b) shows that the titania composition determined through $\mathrm{XRF}$, and the calculated composition based on the amount of precursor are in good agreement. A small deviation is only observed for the MST75 sample.

\subsection{3. ${ }^{29}$ Si NMR}

Fig. 5 shows the ${ }^{29}$ Si NMR spectra of the samples $S T-60$ and $S(c)$. The spectrum of mesoporous silica presents three resonances corresponding to tetrahedral silicate groups of the kinds $\mathrm{Q}^{4}, \mathrm{Q}^{3}$ and $\mathrm{Q}^{2}$. A least-square fitting of three Gaussian functions (dashed lines) enables to calculate the integrated intensities of these resonances, which are directly proportional to the population of the tetrahedral groups. Table 1 indicates the obtained parameters: the average isotropic chemical $\delta_{\mathrm{CS}}$, the full width at half maximum (FWHM) and the $\mathrm{Q}^{\mathrm{n}}$ population. The ${ }^{29} \mathrm{Si}$ spectrum from $S T-60$ shows three resonances partially resolved that can be attributed to the afore-mentioned tetrahedral groups. The resonances maintained the same positions as those of mesoporous silica, though noticeable increments in line-width could be noted. In both samples the width of each resonance is determined by the dispersion of values of the isotropicity. Such depends on the distribution of the chemical and/or structural surroundings around each $\mathrm{Q}^{\mathrm{n}}$ group, due to variations in interatomic distances, bond angles or chemical species interacting with non-bridging oxygen atoms $(N B O)$ of that $\mathrm{Q}^{\mathrm{n}}$. In the
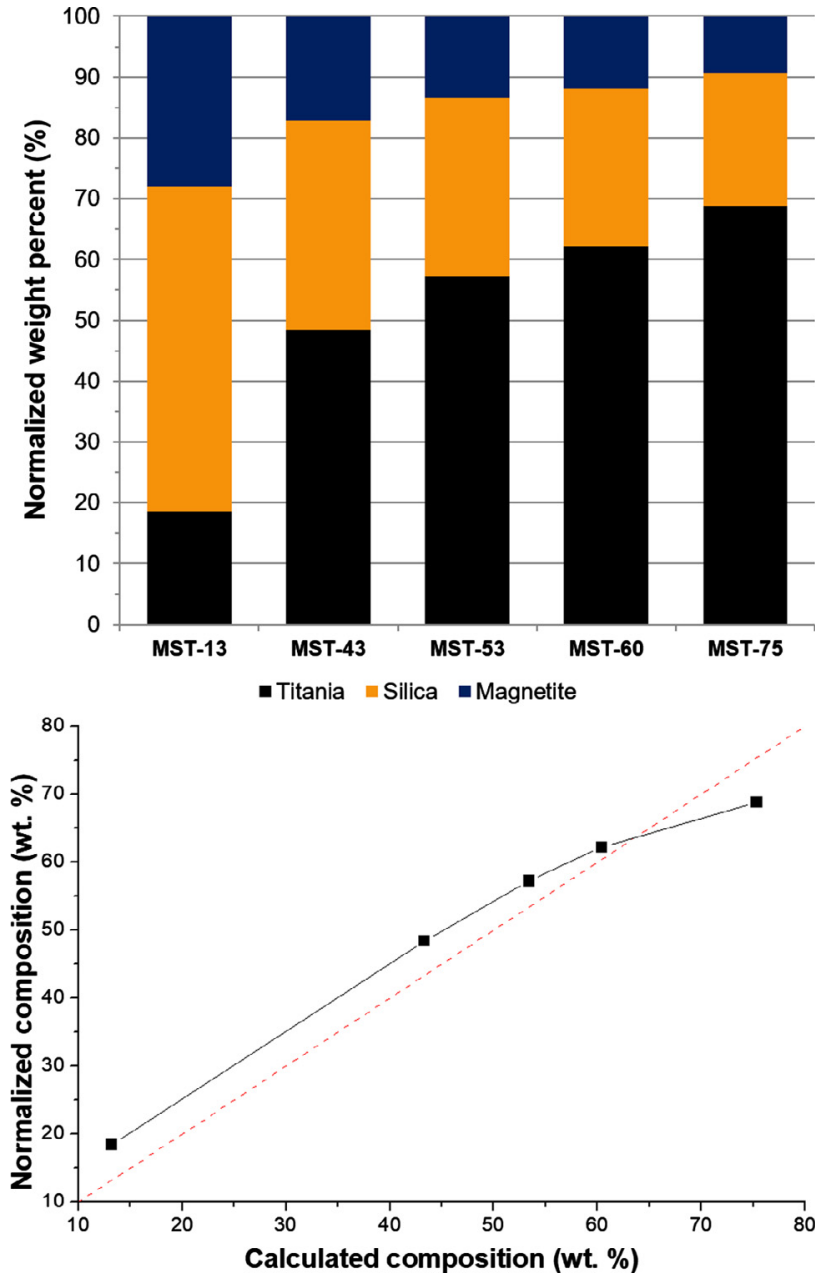

Fig. 4. Normalized chemical composition of the hybrid materials (a) and the relation between the normalized titania composition and the calculated titania composition (b). The dashed red line indicates the estimated titania composition curve (ideal case).

case of the sample $S T-60$, the presence of Ti bonded to NBO causes deviations in the resonance of the ${ }^{29} \mathrm{Si}$ nuclei in these tetrahedrons, resulting in the observed broadening of the spectral lines. Unfortunately, the broadening of the lines does not allow to resolve the resonances of the tetrahedral groups bonded and non-bonded to $\mathrm{Ti}$ atoms, $\mathrm{Q}^{\mathrm{n}}$ and $\mathrm{Q}^{\mathrm{n}}(\mathrm{Ti})$. Therefore, the populations nominally identified as $\mathrm{Q}^{\mathrm{n}}$ in Table 1 contain the contributions of $\mathrm{Q}^{\mathrm{n}}(\mathrm{Ti})$ for the $S T-60$ sample. It is possible to observe that the width of the lines $\mathrm{Q}^{3}$ and $\mathrm{Q}^{4}$ undergoes a broadening effect in virtue of the presence of Ti. The greatest variation in $\delta_{\mathrm{CS}}$ between both samples occurs for $\mathrm{Q}^{4}$, indicating the effect of $\mathrm{Ti}$ as it bonds to $\mathrm{NBO}$ of $\mathrm{Q}^{3}$ tetrahedrons. Analogously, the binding of Ti atoms to $N B O$ of $\mathrm{Q}^{2}$ groups can also be occurring with less likelihood due to the smaller concentration of such species.

\subsubsection{SEM and EDX analyses}

SEM images of various compositions are shown in Fig. 6. Material $M$ (on the top-left), consists of small spherical particles of roughly $10 \mathrm{~nm}$ in diameter, showing a very rough and granular texture. Agglomerates of the particles appear highly porous, potentially favoring homogeneous deposition of the silica over-layer. MCM-41 $[S(c)]$ shown in the middle column (top), consists of well-defined round structures. Such structures are still present in sample $M S(c)$ [third column (top)], suggesting not all silica contributed to coating of the magnetite core. Yet, the intimate interaction between the globular shapes of silica and magnetite, suggests the magnetite may have acted as a "seed" for the 

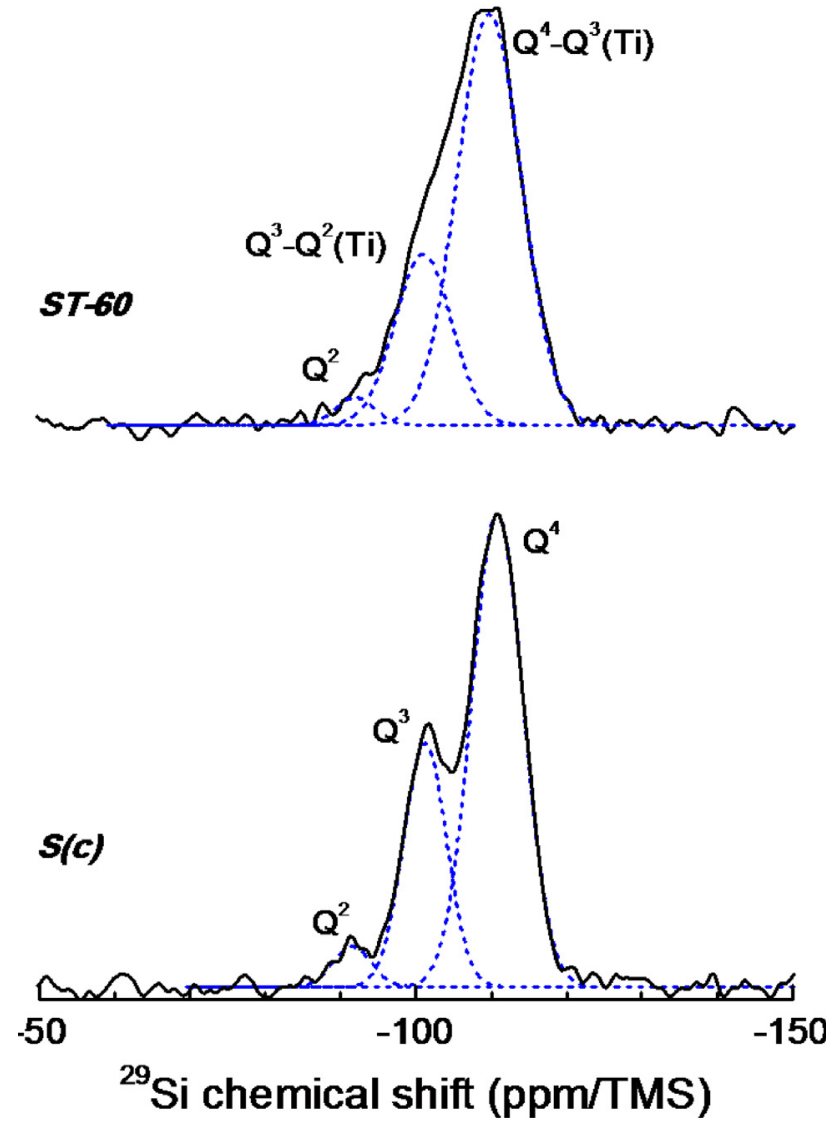

Fig. 5. ${ }^{29} \mathrm{Si}$ NMR spectra of the samples $S T-60$ and $S(c)$.

growth of silica (and thus constitutes a favorable surface for the deposition of silica). The potentially several nanometer thick over-layer cannot be visualized over magnetite texture in the material MS(c). However, the inhibition of phase transformation of magnetite to hematite suggests such layer is present and was confirmed by TEM analysis (Section 3.1.5). We like to note that evolution of gases from the decomposition of the surfactant out of MCM-41 during calcination creates a reducing atmosphere, contributing to the stability of the magnetite core. The size distribution of the precursor materials are illustrated in Fig. S5 of the Supplementary Information.

Images of the titania-coated materials MST-43, MST-53 and MST-60 can be found in the bottom row of Fig. 6 . The applied procedure for $\mathrm{TiO}_{2}$ deposition does not lead to significant changes in texture for the material MST-43 (Fig. 6). For MST-53, interestingly a new texture not previously observed for magnetite and silica begins to appear, which is characterized by quite flat planes, sharp edges and corners. Such new texture might be assigned to titania. In summary, the presented images indicate that as the $\mathrm{TiO}_{2}$ content is increased from MST-43 to MST-60, a growth of titania crystallites may be observed.

EDX analysis of the samples is shown in Fig. 7 (and Fig. S6 of the Supplementary Information), which indicates a homogeneous distribution of all elements $(\mathrm{Fe}, \mathrm{Si}, \mathrm{Ti})$ over all the materials' particles, regardless of the titania composition. The Si signal appears also quite well distributed over the magnetite texture. This supports the presence of a very thin silica layer on these regions, as hypothesized previously, and confirmed by TEM analysis (Section 3.1.5).

\subsubsection{TEM analysis}

To support our TEM investigation on the crystalline constituents of hybrid materials, we used the XRD data (Section 3.1.1) and calculated the interplanar distances $\left(d_{h k l}\right)$ of magnetite and anatase titania phases by applying the Bragg's equation (Eq. (1)) [17].

$n \lambda=2 d_{h k l} \sin \theta$

where $n$ consists of the diffraction order, $d_{h k l}$ represents the interplanar spacing between atomic planes for a given Miller index $(h k l)$, and $\theta$ is half the diffraction angles observed in the XRD analysis (20). The calculated distances were compared to those obtained by the interference fringes of transmission micrographs. All these values can be found in Table 2 along with the respective phase and Miller index.

The transmission images collected for the materials MST-13 (a-e) and MST-60 (f-i) can be found in Fig. 8. The images (a) and (b) refer to different regions of the same particle in sample MST-13 (the analyzed portions are highlighted by the red circles as inset in the respective figures). As previously discussed in the SEM analyses (Section 3.1.4), for materials with titania compositions smaller than $53 \mathrm{wt} \%$ (as in the case of MST-13), the only noticeable textures were those of magnetite and silica. Therefore, the investigated regions for the MST-13 particles were those with either silica [Fig. 8(a)], or magnetite texture [Fig. 8(b)]. In the first case, magnetite agglomerates can be detected as dark spots confined in the interior of silica matrix. The good dispersion of magnetite in the silica phase is important to guarantee an efficient retrieval of the particles from liquid media.

The coating effectiveness over magnetite texture is fundamental to preserve their spinel crystalline structure during calcination, to prevent its photodissolution by the direct interaction with titania phase and also to inhibit the dissolution of the hybrid materials when suspended in acidic media. So the coating of the regions containing magnetite texture with silica is also indispensable for the successful application of the hybrid materials. In Fig. 8(b), it is possible to identify periodic structures deposited on magnetite agglomerates. The observed periodicity is every $\sim 3 \mathrm{~nm}$ [Fig. 8(c)], and cannot be attributed to any crystal planes of magnetite neither titania, since the measured spacing is far greater than the dimensions obtained for any crystal structure. In preliminary tests, low angle X-ray diffraction patterns were collected for the precursor materials $S(c)$ and $M S(c)$ (Fig. S7 of the Supplementary Information), and both showed a diffraction peak at $2 \theta=2.5^{\circ}$. Although consisting of non-crystalline materials, mesoporous silicas tend to be highly organized nanostructures, therefore, their pores may also diffract X-rays to provide information on their arrangement. The corresponding $\theta$ value associated to the low angle $\mathrm{X}$-ray diffraction analysis was inserted in Bragg's equation (Eq. (1)) to obtain the spacing between pores, thus providing $3.5 \mathrm{~nm}$. This value is in good agreement with the spacing observed for the periodic structures seen in Fig. 8(c) for the material MST-13, supporting that they consist of the silica mesoporous structure. With basis on this finding, a representative pore size could be estimated for this material as $2.2 \mathrm{~nm}$ in Fig. 8(d), presenting good agreement with the pore size of mesoporous MCM-41 silica. The observations obtained for the silica coating supports the assumption proposed in Section 3.1.4 about the formation of a thinner silica layer

Table 1

Relative population of $\mathrm{Si}$ species in the samples, as obtained from the integration of the spectra of ${ }^{29} \mathrm{Si}-\mathrm{MAS}$-NMR.

\begin{tabular}{|c|c|c|c|c|c|c|c|c|c|}
\hline \multirow[t]{2}{*}{ Sample } & \multicolumn{3}{|l|}{$\mathrm{Q}^{4}$} & \multicolumn{3}{|l|}{$\mathrm{Q}^{3}$} & \multicolumn{3}{|l|}{$\mathrm{Q}^{2}$} \\
\hline & $\delta_{\mathrm{CS}}(\mathrm{ppm})$ & FWHM (ppm) & Population (\%) & $\delta_{\mathrm{CS}}(\mathrm{ppm})$ & FWHM (ppm) & Population (\%) & $\delta_{\mathrm{CS}}(\mathrm{ppm})$ & FWHM (ppm) & Population (\%) \\
\hline Mesoporous silica & -110.8 & 8.1 & 67 & -101.2 & 6.8 & 29 & -91.5 & 5.8 & 4 \\
\hline$S T-60$ & -109.9 & 10.2 & 70 & -101.0 & 8.9 & 27 & -91.8 & 6.0 & 3 \\
\hline
\end{tabular}




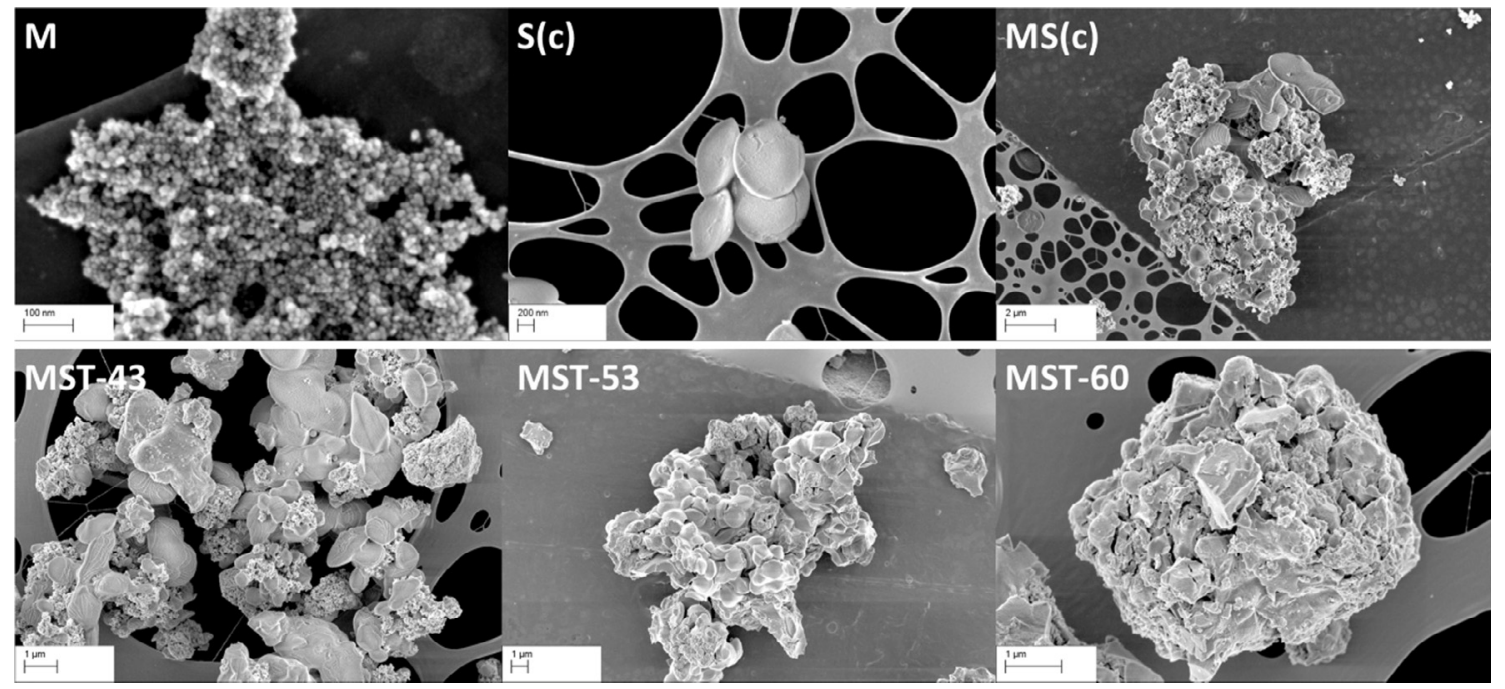

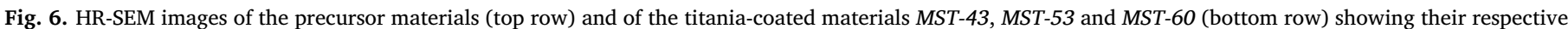
textures.

on the regions presenting magnetite texture.

Yet with regard to the material MST-13, Fig. 8(e) shows planar atomic arrangements (diffraction fringes) for different crystalline phases. By comparing the measured distances in this Fig. with the calculated interplanar distances $\left(d_{h k l}\right)$ in Table 2, crystal phases and Miller indices could be identified. In this figure, we can observe diffraction fringes associated to both magnetite and anatase titania. Interatomic distances of 0.29 and $0.46 \mathrm{~nm}$ correspond to magnetite fringes with Miller indices (2 200$)$ and (1 111 ), respectively.

A characteristic interplanar spacing of $0.36 \mathrm{~nm}$ associated to anatase titania fringes was also identified in this micrograph, and it is related to the Miller index (1 01$)$ of this crystal phase. The demarcated region containing such fringes probably consists of a single titania crystallite with dimensions smaller than $10 \mathrm{~nm}$. Titania crystallites as such were hardly seen for the material MST-13, which reinforces that the titania coating was not effective for this titania composition. It is important to point out that the dimension of $10 \mathrm{~nm}$ for nanosized titania particles is considered the threshold for quantum size effects to take place $[19,20]$. This implies that the smaller the crystallites are, the greater their band gap is, as supposedly would be the case for titania crystallites in the
Table 2

Calculated $d_{h k l}$-spacing for magnetite and titania crystals as a function of the Miller index and the diffraction angle $(\theta)$ obtained by the XRD analysis. Only the $d_{h k l}$-spacing with dimensions related to the measured distances between fringes in the TEM micrographs are shown.

\begin{tabular}{|c|c|c|c|c|}
\hline $\begin{array}{l}\text { Crystalline phase/ } \\
\text { material }\end{array}$ & $\begin{array}{l}\text { Miller } \\
\text { index }\end{array}$ & $\theta\left(^{\circ}\right)$ & $\begin{array}{l}\text { Calculated } d_{h k l^{-}} \\
\text {spacing }(\mathrm{nm})\end{array}$ & $\begin{array}{l}\text { Measured distance } \\
\text { for the fringes }(\mathrm{nm})\end{array}$ \\
\hline Magnetite/ ${ }^{\mathrm{a}}$ & $\left(\begin{array}{llll}1 & 1 & 1\end{array}\right)^{\mathrm{a}}$ & $9.18^{\mathrm{a}}$ & $0.481^{\mathrm{a}}$ & 0.46 \\
\hline Magnetite $/ M$ & $(220)$ & 15.3 & 0.288 & 0.29 \\
\hline Titania/MST-60 & $\left(\begin{array}{lll}1 & 0 & 1\end{array}\right)$ & 12.8 & 0.344 & 0.36 \\
\hline
\end{tabular}

\footnotetext{
a Despite not observed by our XRD analysis for any material, this orientation
} is reported for magnetite [18].

material MST-13. Thus, they would require radiation with shorter wavelengths to be photoexcited. The alteration in the band gap and the surface crystallite density may have a serious influence on the performance of low-titania-content materials, as it will be discussed in Section 3.2.

Periodic structures presenting $0.60 \mathrm{~nm}$ spacing were commonly
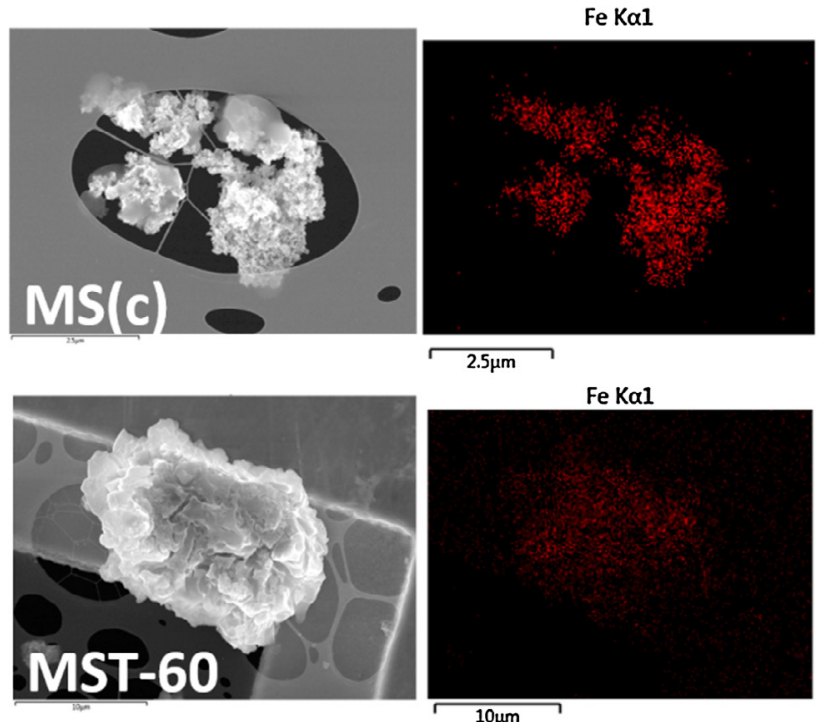

Si Kal

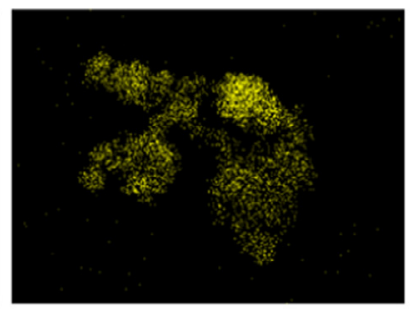

Si Kal

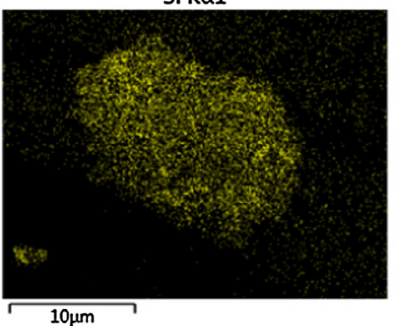

Ti Kal

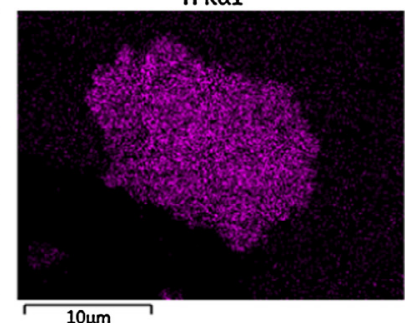

Fig. 7. EDX analysis of MS(c) (top row) and MST-60 (bottom row) showing their elemental distribution. HR-SEM images of their respective EDX mapping images are shown at the very left for comparison. 

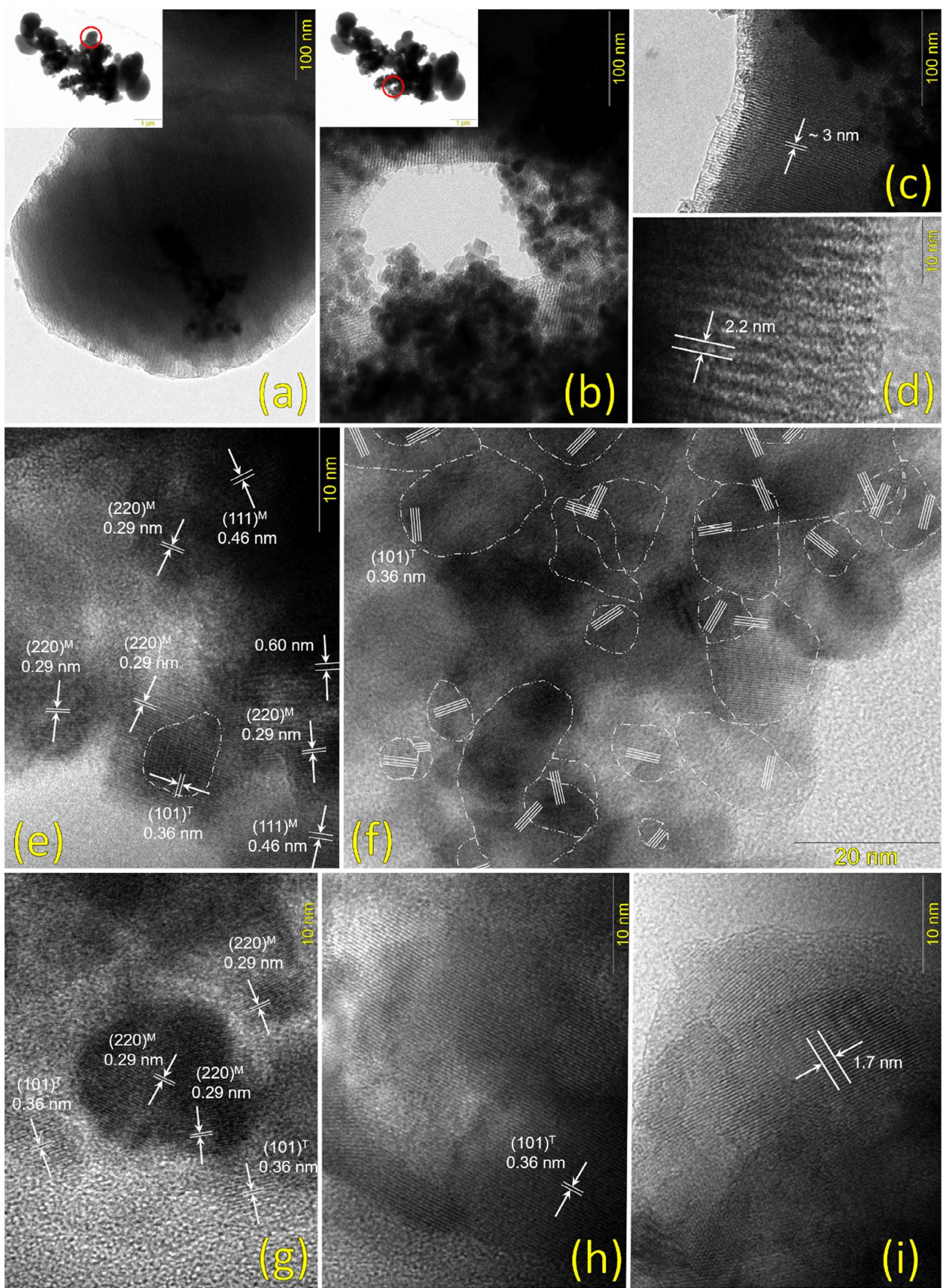

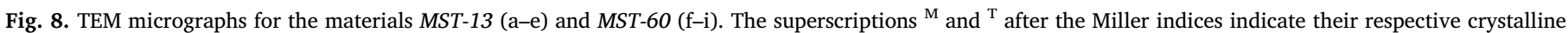
phase (magnetite or titania).

observed in both MST-13 and MST-60 [also seen in Fig. 8(e)], nonetheless, they could not be attributed to any of the constituent phases.

With respect to the material MST-60, Fig. 8(f) shows many demarcated regions in which fringes present a characteristic anatase interplanar distance of $0.36 \mathrm{~nm}$. In this micrograph, it is not possible to find atomic planes related to magnetite, therefore anatase titania is far 


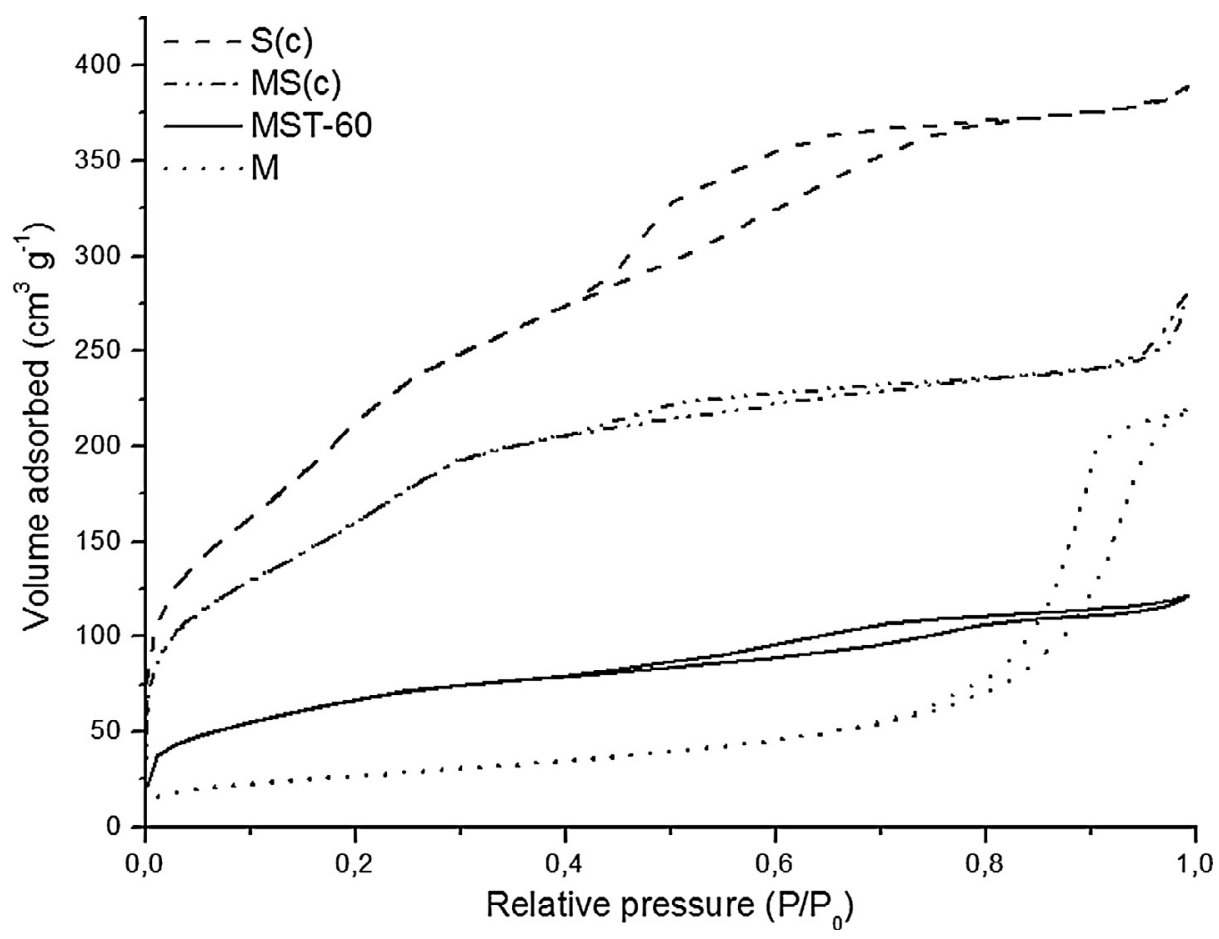

Fig. 9. Nitrogen adsorption isotherms for the single precursor materials $S(c)$ and $M$, for the hybrid precursor material $M S(c)$, and for the material $M S T-60$.

more predominant than magnetite in this material. Considering that many of the demarcated regions likely correspond to anatase titania crystallites, we note that this material contains considerably more of such crystallites, which also tended to be greater for this titania composition. Similarly to what was observed for the material MST-13, regions in which magnetite and titania phases coexisted were also found for the material MST-60, as can be seen in Fig. 8(g), nonetheless, the visualization of magnetite fringes was far more difficult.

Titania crystallites with dimensions far greater than those seen in Fig. 8(f) were also found for the material MST-60, and Fig. 8(h) shows an example of such crystallite. We can note that a single titania fringe nearly occupies the entire micrograph in this figure.

Finally, in Fig. 8(i), a periodic channel structure such as that of silica could also be visualized for the material MST-60. Although titania is apparently coating this silica structure, the collected images did not allow us to observe if the titania deposition inside the pores was effective.

\subsubsection{Surface area measurements}

Isotherms for the materials $M, S(c), M S(c)$, and $M S T-60$ can be found in Fig. 9. All the samples show a typical type IV isotherm, being the hysteresis loop an indicative for inter-particle pores [21]. Type IV isotherms are commonly related to high surface area mesoporous materials, such as mesoporous silicas [likewise $S(c)$ ], with pore diameters in the range between 2 and $50 \mathrm{~nm}$. For $M S(c)$, the hysteresis loop at high $\mathrm{P} / \mathrm{P}_{0}$ is less pronounced, suggesting the $M$ particles have less interparticle voids when coated with a silica overlayer. For MST-60, titania clearly decreases the overall surface area, but does not completely block the access of nitrogen to the mesopores of silica, since mesoporosity can be also noted for such material.

The respective surface areas of the afore-mentioned materials can be found in Table 3. As evident from the isotherms, the specific surface area of the material $M S(c)$ presents an intermediate value between those of the materials $M$ and $S(c)$ representing the weight-average of the surface area of $M$ and $S(c)$. The incorporation of titania led to a further reduction in the surface area for the material $M S T-60$, in agreement with the lower weight-percentage of the mesoporous silica $S$ (c). Despite the decrease in the average surface area of the hybrid
Table 3

Surface area measurements data obtained for the individual and hybrid materials.

\begin{tabular}{ll}
\hline Material & BET surface area $\left(\mathrm{m}^{2} \mathrm{~g}^{-1}\right)$ \\
\hline$M$ & $97.50( \pm 0.10)$ \\
$S(c)$ & $811( \pm 21)$ \\
$M S(c)$ & $589.1( \pm 6.7)$ \\
$M S T-60$ & $247.8( \pm 1.2)$ \\
\hline
\end{tabular}

material, its specific surface area is greater than most commercially available titanium dioxide types.

\subsection{Photodegradation experiments}

In this study, $\mathrm{pH}$ values of $\sim 3.0$ and $\sim 7.2$ were selected for evaluating the performance of the catalyst materials in conversion of 4-CP, and the data collected were used to construct Fig. 10. We verified the materials were not active in dark conditions (Fig. 10 top), and degradation of 4-CP did not occur for solutions exposed to UV without any of the composites (Fig. S8 of the Supplementary Information).

Comparing the activity data shown in Fig. 10, three observations can be made: (i) the samples containing relatively low quantities of $\mathrm{TiO}_{\mathrm{x}}$ do not show significant degradation of 4-CP, (ii) increasing the quantity of $\mathrm{TiO}_{\mathrm{x}}$ above the threshold value of $53 \mathrm{wt} \%$ does not significantly improve performance (compare MST-53, MST-60 and MST75), and (iii) the $\mathrm{pH}$ has little effect on the rate of degradation of 4-CP.

The necessity of a minimum of $53 \mathrm{wt} \% \mathrm{TiO}_{2}$ suggests that crystalline Anatase is required to induce photocatalytic activity of the composites, which has been identified in the active samples by SEM, XRD and Raman analysis. It is well-known that at relatively low loading of $\mathrm{TiO}_{2}$, grafting on siliceous substrates such as MCM-41 leads to isolated and polymeric $\mathrm{TiO}_{\mathrm{x}}$ species. These species require significantly shorter wavelengths than crystalline Anatase to be photo-excited [22], likely shorter than provided by the applied illumination source in the present study $(\sim 360 \mathrm{~nm})$. It should be noted that the Anatase phase was also detected for the material MST-43 through both Raman spectroscopy 

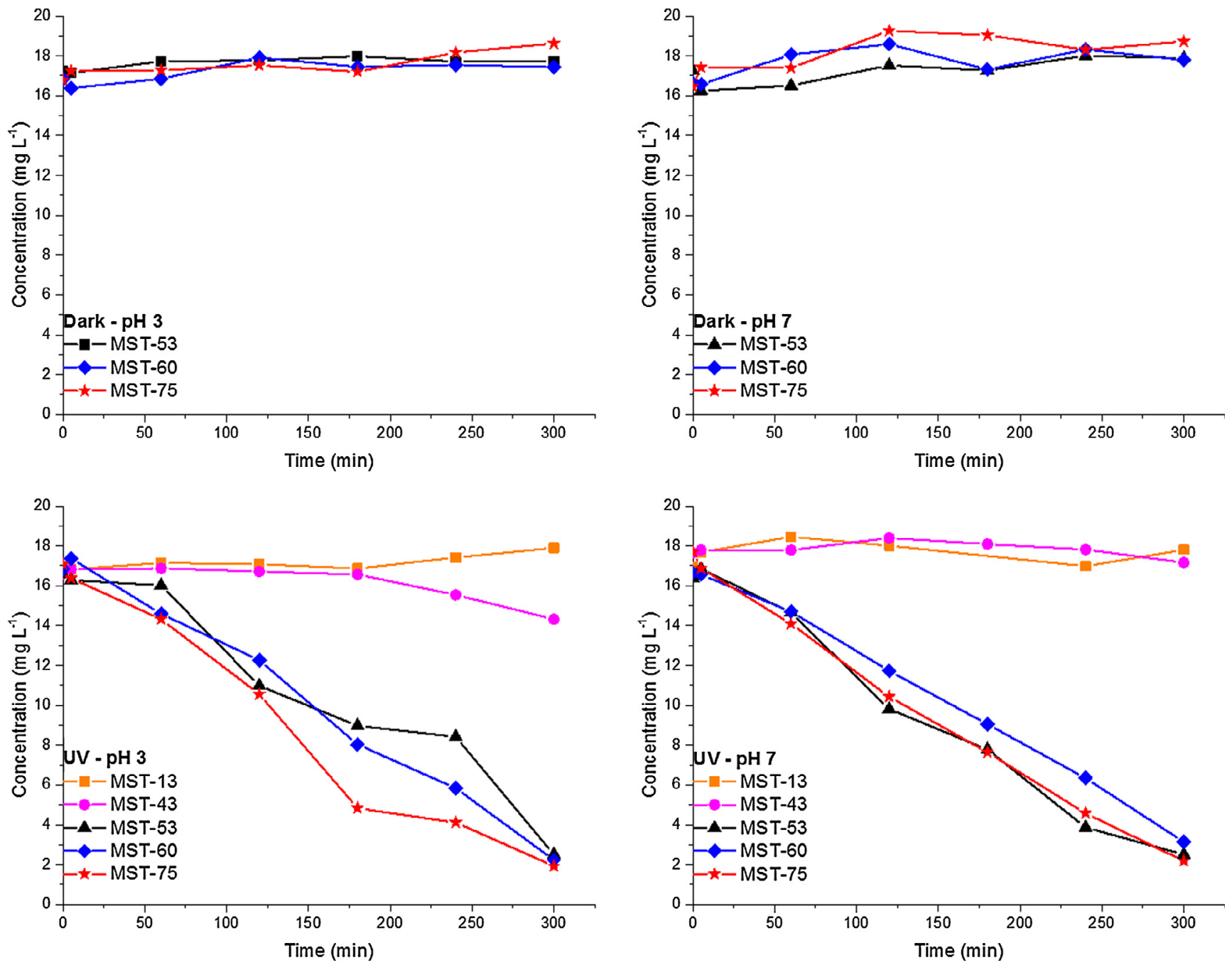

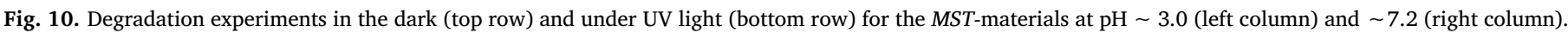
$\mathrm{pH}$ conditions and dark/UV regimes are specified above the inset legends. Catalyst mass equals $15.0 \mathrm{mg}$.

and XRD (as seen in the Section 3.1.1), and for MST-13 through TEM analysis (Section 3.1.5), while these materials present a very poor photoactivity. As evidenced in TEM analysis (Section 3.1.5), titania crystallites present in MST-13 (and likely also in MST-43) are that small, that quantum size effects result in required UV absorption wavelengths smaller than provided by the UV illumination used in the present study, similar to isolated and polymeric $\mathrm{TiO}_{\mathrm{x}}$ species. The low crystallite surface density [also discussed in the TEM analysis (Section 3.1.5)] may also have compromised significantly the efficiency for such low-titaniacontent materials.

The small difference in performance by increasing the $\mathrm{TiO}_{\mathrm{x}}$ content from $53 \mathrm{wt} \%$ to $75 \mathrm{wt} \%$, is likely the consequence of increasing growth of the crystallites, and therefore the relatively insignificant increase in active $\mathrm{TiO}_{\mathrm{x}}$ surface by increasing the $\mathrm{TiO}_{\mathrm{x}}$ content.

Concerning the similar photocatalytic degradation performance at both studied $\mathrm{pH}$ values, the linear behavior suggests the degradation rate is independent of 4-CP concentration, and the degradation obeys zero-order kinetics. The dark experiments (top row) are in agreement with this observation, showing that the concentration of 4-CP remains nearly constant for all the studied materials, which indicates that adsorption of 4-CP is unfavorable. Contrary to often reported in the literature [20], adsorption of the substrate apparently does not play an essential role in the degradation mechanism. This observation is supported by other works that report the degradation of organic compounds by $\mathrm{TiO}_{2}$ without the occurrence of adsorption [23]. 4-CP is known for being poorly adsorbed on $\mathrm{TiO}_{2}$ surfaces, which is explained by the displacement of 4-CP adsorption equilibrium by water molecules in virtue of their greater affinity for theTiO ${ }_{2}$ adsorption sites [24]. It is also described that 4-CP degradation can take at least three routes based on the hydroxyl-mediated mechanisms to produce mainly hydroquinone $(H Q)$, 4-catechol (4-CC) and non-aromatic intermediates $[20,25]$. After the conversion to $H Q$ and 4-CC, these compounds may undergo adsorption on the surface to be further mineralized. We propose reactive oxygen species formed on the surface of the catalysts might desorb into solution, thus triggering the formation of $H Q$ and 4$C C$ in the medium to be posteriorly adsorbed and mineralized on the $\mathrm{TiO}_{2}$ surface. This observation is in alignment with the work of Choi et al. [26], which provided very strong evidence of the desorption of reactive oxygen species from $\mathrm{TiO}_{2}$ in aqueous medium. Finally, the absence of sorption-dominated conversion is in agreement with the similar activity observed in acidic and neutral $\mathrm{pH}$, which likely changes the relative surface charge of $\mathrm{TiO}_{2}$.

To uncover the photodegradation effect on the $\mathrm{pH}$ of the media, the final $\mathrm{pH}$ was also measured for the suspensions of the sample MST-60. A drastic $\mathrm{pH}$ decay was observed for the suspension with initial $\mathrm{pH} \sim 7.2$, which reached final $\mathrm{pH} \sim 4.2$. For the suspension with initial $\mathrm{pH} \sim 3.0$, the final $\mathrm{pH}$ was slightly reduced to $\sim 2.9$. The acidification of the media is not surprising because the decomposition of 4-CP generates $\mathrm{HCl}$ in solution. This also implies that 4-CP structure is not altered during the degradation process strictly due to $\mathrm{pH}$ influence, since the 


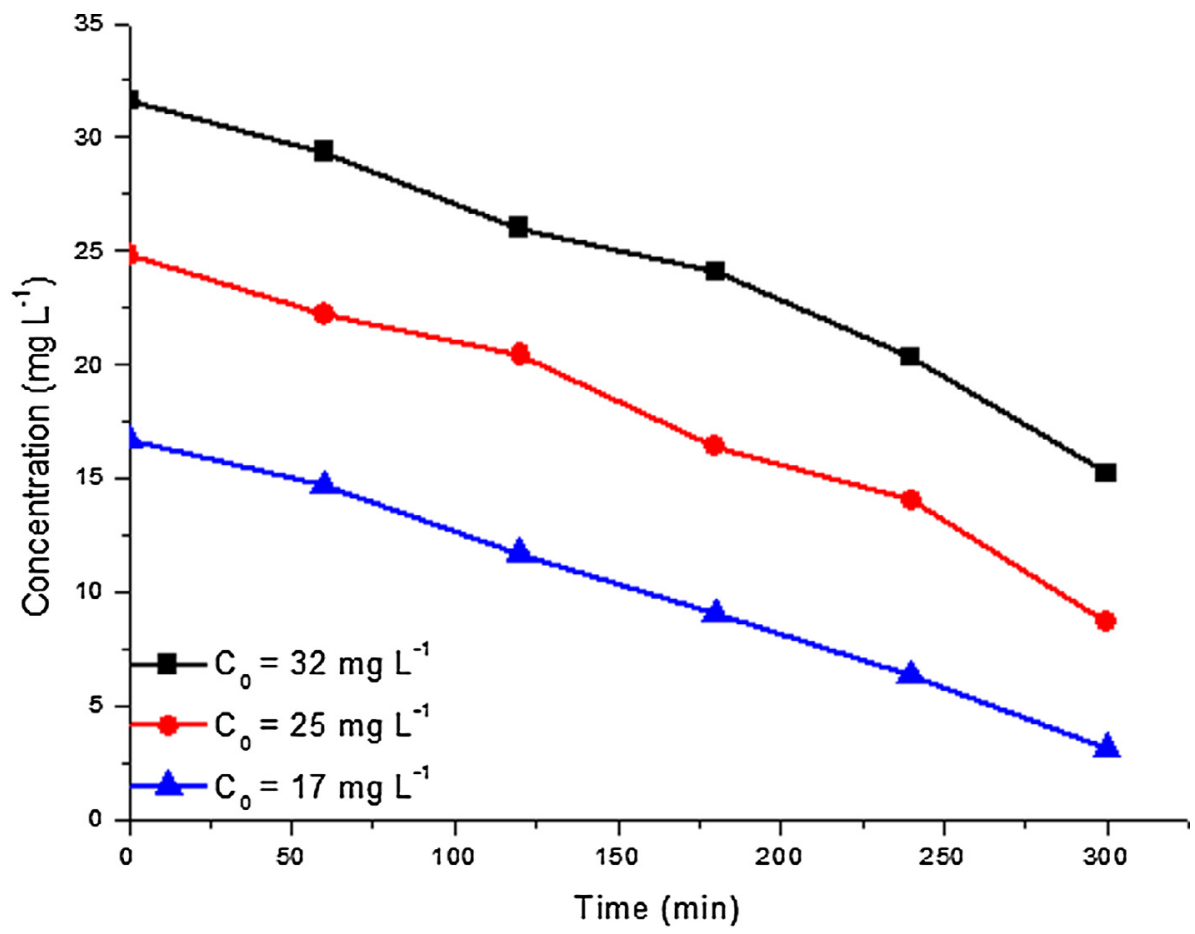

(a)

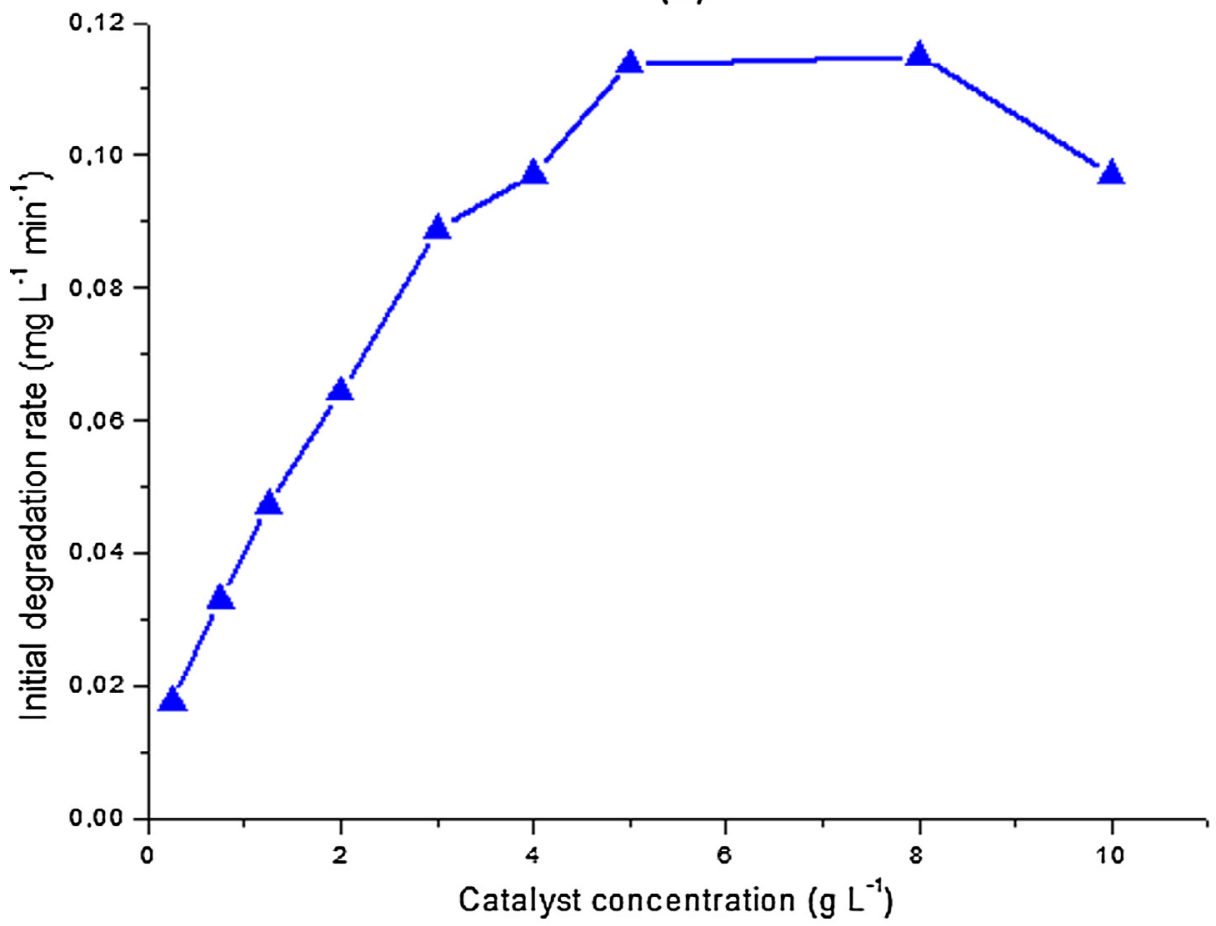

(b)

Fig. 11. Photodegradation as a function of the 4-CP concentration (catalyst mass $=15.0 \mathrm{mg} ; \mathrm{pH} \sim 7.2$ ) (a), and the initial degradation rate as a function of the catalyst concentration for MST-60 (b).

$\mathrm{pK}_{\mathrm{a}}$ of 4-CP is 9.4, and significant deprotonation effects only occur at $\mathrm{pH} \geq \mathrm{pK}_{\mathrm{a}}(4-\mathrm{CP})-2$ (more explicitly, $\geq 7.4$ ). It is also important to point out that the variation in the $\mathrm{pH}$ of the media may also be related to the similar results provided for the experiments with different initial $\mathrm{pH}$, since during the degradation process, the media will be found acidified at some degree in both cases.

At the end of the degradation experiments, the supernatants of the suspensions with initial pH 3.0 and 7.2 were evaluated through ICP-OES to ascertain the occurrence of the decomposition of the hybrid particles after the degradation process, if any, and the material MST- 60 was used as a reference. Solutions of each $\mathrm{pH}$ without any material were also tested under the same conditions to serve as control solutions. The concentrations of $\mathrm{Ti}, \mathrm{Si}$ and $\mathrm{Fe}$ were below the detection limit for both initial $\mathrm{pH}$ conditions, thus indicating that the hybrid materials present satisfactory cohesion to withstand the chemical and physical events inside the reactors. Similarly, the evaluated elements were not detected 
for the control solutions.

\subsubsection{Effect of 4-CP and catalyst concentrations on degradation rate}

Fig. 11(a) shows the effect of the initial 4-CP concentration on the degradation kinetics. Independent of the initial concentration of 4-CP, the degradation curves demonstrates zero-order kinetics, again suggesting adsorption of 4-CP is not dominant in determining the degradation rate. Fig. 11(b) shows the effect of catalyst concentration on the obtained reaction rate. The initially rising activity as a function of increasing concentration is quite typical for photocatalytic slurry reactors. A maximum efficiency is achieved for a catalyst concentration between 5.00 and $8.00 \mathrm{~g} \mathrm{~L}^{-1}$. Initially, increasing catalyst concentration is required to achieve sufficient light absorption in the top-illuminated reactor. Once effective absorption has been achieved $\left(\geq 8 \mathrm{~g} \mathrm{~L}^{-1}\right)$, the scattering and absorption of incident light by the suspended particles start to compromise the illumination of other particles in the medium, especially the ones at the end of the optical path. At this point, the generation of reactive oxygen species is hardly affected, and the efficiency in the range from 8 to $10 \mathrm{~g} \mathrm{~L}^{-1}$ rather constant.

When the performance of MST-60 is compared to other commercially available photocatalysts (P25 and Hombikat, see Fig. S9 of the Supplementary Information), the performance is generally lower (on a per gram basis). The activity of titania deposited directly on Magnetite (MT-60) is also very low (not shown), likely due to the absence of Anatase, as shown in XRD diffractogram (see Section 3.1.1 and Fig. S2 of the Supplementary Information), which suggests insufficient $\mathrm{TiO}_{\mathrm{x}}$ is directly deposited on Magnetite. This shows the silica layer is critical for efficient growth of titania crystallites on magnetite, applying the proposed titania coating route. Given the above absence of activity of this weight fraction of the composite, it is not surprising the performance per gram is relatively low as compared to pure $\mathrm{TiO}_{2}$ samples.

Finally, it should be noted that ST-60 outperforms MST-60 (see also Fig. S9 of the Supplementary Information), which besides to the aforementioned inactive weight fraction of magnetite, could be attributed to unfavorable light absorption and charge recombination processes induced by the Magnetite.

The application of photocatalysts in fixed bed and slurry reactors is affected by many practical parameters. A photodegradation process undertaken in fixed-bed reactors demonstrates to be more feasible, but yet suffers from several drawbacks, such as low surface area to volume ratios and inefficiencies related to light absorption and scattering in the reactor [20]. Slurry systems, by their turn, consist of fine solid catalyst particles highly dispersed in a liquid phase. The medium is very turbulent due to the agitation provided by either mechanical stirring or gas bubbling. Besides attributing better surface area to volume ratios, slurry systems also favor mass transfer phenomena between the catalyst and the liquid medium through substrate adsorption and diffusion processes. Nevertheless, slurry reactors are claimed to be impractical for photodegradation processes in scaled-up reactors due to difficulties in separating the photocatalyst $[4,6-10]$. The production of a magnetic photocatalyst could perhaps allow circumventing the drawbacks associated to slurry systems and turn the recovery of the photocatalyst more practical.

\section{Conclusions}

A magnetically-extractable hybrid of magnetite, MCM-41 silica and titania (Anatase phase) was successfully synthesized and applied as a photocatalyst. The coating step of $\mathrm{TiO}_{2}$ using boiling ethanol solution of titanium isopropoxide (TIP) resulted in a good correlation between the calculated and the measured amounts of titania. Up to a $\mathrm{TiO}_{2}$ content of $48.3 \mathrm{wt} \%$, grafting results in low density of $\mathrm{TiO}_{2}$ crystallites on the silica (MCM-41) surface, leading to poor photoactivity. The material was demonstrated to be chemically stable in acidic and neutral $\mathrm{pH}$ and to be a poor adsorbent for 4-chlorophenol, which suggests that the degradation mechanism is related to production of reactive oxygen species and to the transient contact between the catalyst surface and the substrate. Photocatalytic activity was only observed for titania compositions $\geq 57.2 \mathrm{wt} \%$. $\mathrm{TiO}_{2}$ crystallites are inducing the photocatalytic activity: absorption of UV light of relatively long wavelengths (in the wavelength range of $360-380 \mathrm{~nm}$ ) by isolated and polymeric $\mathrm{TiO}_{\mathrm{x}}$ is not feasible. Increasing the $\mathrm{TiO}_{2}$ loading to values above $57.2 \mathrm{wt} \%$ hardly improved the performance in catalyst concentrations from 5 to $10 \mathrm{~g} \mathrm{~L}^{-1}$. The degradation of 4-chlorophenol at $\mathrm{pH} 3.0$ and 7.2 followed zero-order kinetics, confirming the mechanism of 4-chlorophenol degradation involves (desorbed) reactive oxygen species. Although the performance of the hybrid material (on a $\mathrm{g}^{-1}$ basis) underperforms Anatase or P25 $\mathrm{TiO}_{2}$, the proven easy separation of the catalyst from purified liquid by applying a magnetic field favors the composite over $\mathrm{TiO}_{2}$ for practical application in waste water remediation.

\section{Acknowledgements}

The authors thank the Centro de Microscopia Eletrônica of the Institute of Biosciences of Unesp in Botucatu (Brazil) for preliminary scanning electron imaging, the University of Twente and the Photocatalytic Synthesis Group (PCS group) (the Netherlands) for the internship and all support granted to the first author, the MESA + and the Catalytic Processes and Materials Group (CPM group) (the Netherlands) for all the technical support. This work was supported by Fapesp [grant numbers 2015/05224-0, 2013/22955-2]; and CNPq [grant number 120112/2013-2).

\section{Appendix A. Supplementary material}

Supplementary data associated with this article can be found, in the online version, at https://doi.org/10.1016/j.apsusc.2018.06.218.

\section{References}

[1] C. Baird, M. Cann, Química Ambiental, fourth ed., Bookmann, Porto Alegre, 2011.

[2] S.E. Manahan, Environmental Science and Technology, Lewis Publishers, Boca Raton, 1997.

[3] V.M. Cristante, S.M.A. Jorge, J.P.S. Valente, M.J. Saeki, A.O. Florentino, P.M. Padilha, $\mathrm{TiO}_{2}$ films organofunctionalized with 2-aminothiazole ligand and adsorbed Pd(II) ions applied in the photocatalytic degradation of phenol in an aqueous medium, Thin Solid Films 515 (2007) 5334-5340, http://dx.doi.org/10 1016/j.tsf.2007.01.023.

[4] S.M. Gupta, M. Tripathi, A review of $\mathrm{TiO}_{2}$ nanoparticles, Chin. Sci. Bull. 56 (2011) 1639, http://dx.doi.org/10.1007/s11434-011-4476-1.

[5] S. Ahmed, M.G. Rasul, R. Brown, M.A. Hashib, Influence of parameters on the heterogeneous photocatalytic degradation of pesticides and phenolic contaminants in wastewater: a short review, J. Environ. Manage. 92 (2011) 311-330, http://dx. doi.org/10.1016/j.jenvman.2010.08.028.

[6] H. Liu, Z. Jia, S. Ji, Y. Zheng, M. Li, H. Yang, Synthesis of TiO2/SiO2@Fe3O4 magnetic microspheres and their properties of photocatalytic degradation dyestuff, Catal. Today 175 (2011) 293-298, http://dx.doi.org/10.1016/j.cattod.2011.04. 042.

[7] A.A. Aziz, C.K. Cheng, S. Ibrahim, M. Matheswaran, P. Saravanan, Visible light improved, photocatalytic activity of magnetically separable titania nanocomposite, Chem. Eng. J. 183 (2012) 349-356, http://dx.doi.org/10.1016/j.cej.2012.01.006.

[8] C. Wang, L. Yin, L. Zhang, L. Kang, X. Wang, R. Gao, Magnetic ( $\gamma$-Fe2O3@SiO2) n@TiO2 functional hybrid nanoparticles with actived photocatalytic ability, J. Phys. Chem. C 113 (2009) 4008-4011, http://dx.doi.org/10.1021/jp809835a.

[9] D.G. Shchukin, A.I. Kulak, D.V. Sviridov, Magnetic photocatalysts of the core-shell type, Photochem. Photobiol. Sci. 1 (2002) 742-744, http://dx.doi.org/10.1039/ B207477J.

[10] Y.S. Chung, S. Bin Park, D.-W. Kang, Magnetically separable titania-coated nickel ferrite photocatalyst, Mater. Chem. Phys. 86 (2004) 375-381, http://dx.doi.org/10. 1016/j.matchemphys.2004.03.027.

[11] S. Lee, J. Drwiega, D. Mazyck, C.-Y. Wu, W.M. Sigmund, Synthesis and characterization of hard magnetic composite photocatalyst-barium ferrite/silica/titania, Mater. Chem. Phys. 96 (2006) 483-488, http://dx.doi.org/10.1016/j. matchemphys.2005.07.039.

[12] J.P. Cheng, R. Ma, M. Li, J.S. Wu, F. Liu, X.B. Zhang, Anatase nanocrystals coating on silica-coated magnetite: role of polyacrylic acid treatment and its photocatalytic properties, Chem. Eng. J. 210 (2012) 80-86, http://dx.doi.org/10.1016/j.cej.2012. 08.059.

[13] S.C. Pang, S.Y. Kho, S.F. Chin, Fabrication of magnetite/silica/titania core-shell nanoparticles, J. Nanomater. 2012 (2012) 1-6.

[14] A. Walcarius, L. Mercier, Mesoporous organosilica adsorbents: nanoengineered 
materials for removal of organic and inorganic pollutants, J. Mater. Chem. 20 (2010) 4478-4511, http://dx.doi.org/10.1039/B924316J.

[15] H.E. Ghandoor, H.M. Zidan, M.M.H. Khalil, M.I.M. Ismail, Synthesis and some physical properties of magnetite $\left(\mathrm{Fe}_{3} \mathrm{O}_{4}\right)$ nanoparticles, Int. J. Electrochem. Sci. 7 (2012) 5734-5745.

[16] U.S. Secretary of Commerce on behalf of the United States of America, NIST Livro de Química na Web, SRD 69, 2017. < https://webbook.nist.gov/cgi/cbook.cgi? ID $=$ C13463677\&Units $=$ SI\&Type $=$ IR-SPEC $>$.

[17] D.B. Williams, C.B. Carter, Transmission Electron Microscopy: A Textbook for Materials Science, second ed., Springer, New York, 2009.

[18] W. Lu, M. Ling, M. Jia, P. Huang, C. Li, B. Yan, Facile synthesis and characterization of polyethylenimine-coated $\mathrm{Fe}_{3} \mathrm{O}_{4}$ superparamagnetic nanoparticles for cancer cell separation, Mol. Med. Rep. (2014) 1080-1084, http://dx.doi.org/10.3892/mmr. 2014.1906.

[19] K.D. Dubois, G. Li, Innovative photocatalysts for solar fuel generation by $\mathrm{CO}_{2}$ reduction, New Futur. Dev. Catal. Elsevier, 2013, pp. 219-241, http://dx.doi.org/10. 1016/B978-0-444-53872-7.00010-8.

[20] M.R. Hoffmann, S.T. Martin, W. Choi, D.W. Bahnemann, Environmental applications of semiconductor photocatalysis, Chem. Rev. 95 (1995) 69-96, http://dx.doi. org $/ 10.1021 / \mathrm{cr} 00033 \mathrm{a} 004$.

[21] K.S.W. Sing, D.H. Everett, R.A.W. Haul, L. Moscou, R.A. Pierotti, J. Rouquerol,
T. Siemieniewska, Reporting physisorption data for gas/solid systems, Handb. Heterog. Catal. American Cancer Society, 2008, pp. 1217-1230, http://dx.doi.org/ 10.1002/9783527610044.hetcat0065.

[22] K. Cendrowski, X. Chen, B. Zielinska, R.J. Kalenczuk, M.H. Rümmeli, B. Büchner, R. Klingeler, E. Borowiak-Palen, Synthesis, characterization, and photocatalytic properties of core/shell mesoporous silica nanospheres supporting nanocrystalline titania, J. Nanoparticle Res. 13 (2011) 5899-5908, http://dx.doi.org/10.1007/ s11051-011-0307-1.

[23] J.S. Romão, M.S. Hamdy, G. Mul, J. Baltrusaitis, Photocatalytic decomposition of cortisone acetate in aqueous solution, J. Hazard. Mater. 282 (2015) 208-215, http://dx.doi.org/10.1016/j.jhazmat.2014.05.087.

[24] U. Stafford, K.A. Gray, P.V. Kamat, Radiolytic and $\mathrm{TiO}_{2}$-assisted photocatalytic degradation of 4-chlorophenol. A comparative study, J. Phys. Chem. 98 (1994) 6343-6351, http://dx.doi.org/10.1021/j100076a019.

[25] X. Li, J.W. Cubbage, T.A. Tetzlaff, W.S. Jenks, Photocatalytic degradation of 4chlorophenol. 1. The hydroquinone pathway, J. Org. Chem. 64 (1999) 8509-8524, http://dx.doi.org/10.1021/jo990820y.

[26] W. Choi, S. Kim, S. Cho, H.-I. Yoo, M.-H. Kim, Photocatalytic reactivity and diffusing $\mathrm{OH}$ radicals in the reaction medium containing $\mathrm{TiO}_{2}$ particles, Kor. J. Chem. Eng. 18 (2001) 898-902, http://dx.doi.org/10.1007/BF02705615. 